\title{
Buckling and Torsional Instabilities of a Nanoscale Biological Rope Bound to an Elastic Substrate
}

Chris Peacock, Eva Lee, Theo Beral, Richard Cisek,

Danielle Tokarz and Laurent Kreplak

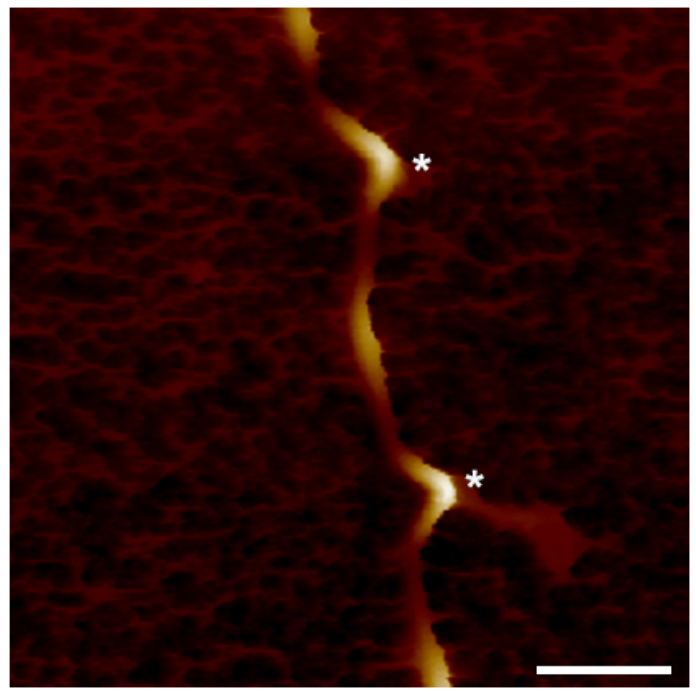

$730 \mathrm{~nm}$

S 1: AFM height image of an LDE tendon fibril on a $25 \%$ pre-strained PDMS substrate after release. The out-of-plane sinewave buckling mode is clearly visible as well as two sites of loop formation indicated by asterisks. The bar is $1 \mu \mathrm{m}$. 

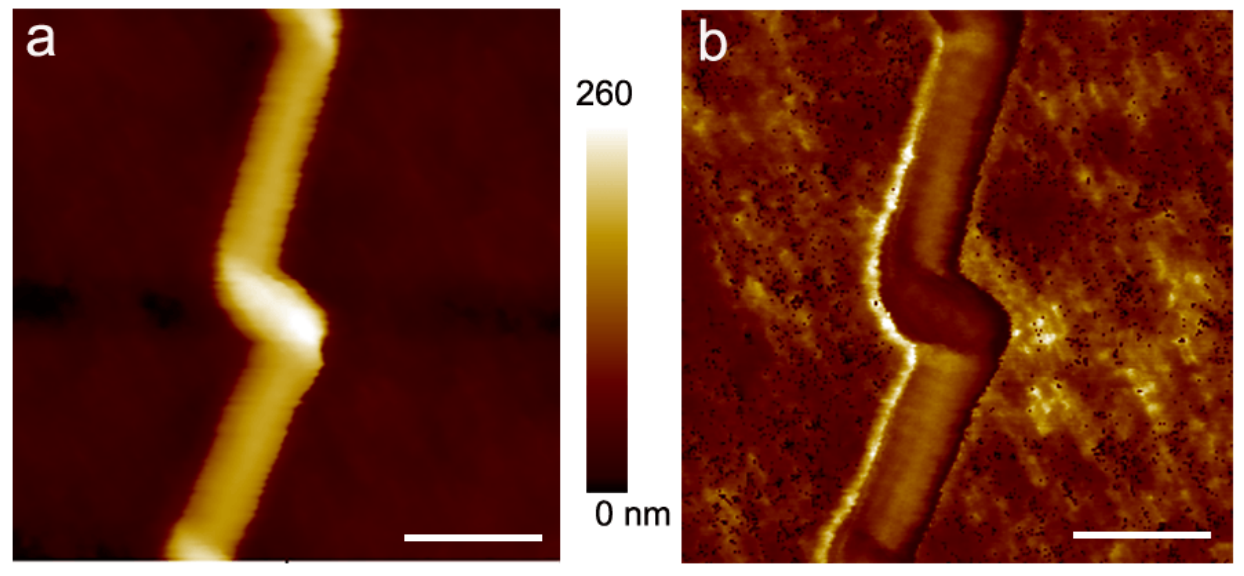

16

S 2: Swelling of the birdcages is associated with a decrease in indentation modulus (a) Height image of a triton treated DDF fibril on a $30 \%$ pre-strained PDMS covered with PBS. The birdcage in the middle of the image is taller than the surrounding fibril segments. The bar is $500 \mathrm{~nm}$. (b) Indentation modulus image computed using the Sneddon model, with a Poisson ratio of 0.5, a half-cone angle of $18^{\circ}$, and a fitting range between 1 and $8 \mathrm{nN}$. The birdcage in the middle of the image is softer than the surrounding fibril segments. The bar is $500 \mathrm{~nm}$.

Movie S3: Movie of the SHG intensity of the fibril in Figure 5 a as linear polarization is varied over $180^{\circ}$. Each of the 8 frames was contrast enhanced using ImageJ, the intensity of each frame was equalized and normalized. The frame rate is 0.25 fps. 